


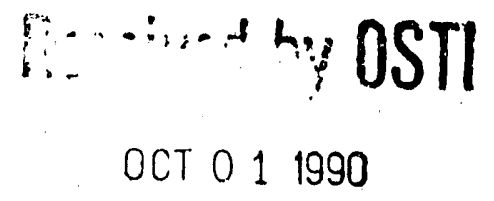

DOE/NBM- -1070

DE91 000017

\section{Solid Waste \\ Projection Model System Overview}

Prepared for

Westinghouse Hanford Company by Pacific Northwest Laboratory

Richland, Washington

August 1950 
This overview was prepared for Westinghouse Hanford Company by Pacific Northwest Laboratory, Richland, Washington, under contract DE-AC06-RLO 1830 with the U.S. Department of Energy. The Pacific Northwest Laboratory is operated for the U.S. Department of Energy by Battelle Memorial Institute.

Available as

DOENBM-1070

from the

Office of Scientific and Technical Information

Oak Ridge, Tennessee

This report was prepared as an account of work sponsored by the United States Government. Neither the United States nor the U.S. Department of Energy, nor any of their employees, or any of their contractors, subcontractors, or their employees, makes any warranty, express or implied, or assumes any legal liability or responsibility for the accuracy, completeness, or usefulness of any information, apparatus, product, or process disclosed, or represents that its use would not infringe privately owned rights. 


\section{Solid Waste Projection Model System Overview}

Waste management is becoming an inc.easingly important topic. Management of current and future solid wastes will require the development of appropriate treatment, storage, and disposal (TSD) facilities and operations to match the characteristics of the wastes. Inadequate provision of these $\mathrm{TSD}^{(\mathbf{a})}$ facilities and operations will surely incur costs in the form of agency fines or excessive handling fees and may even result in the need to curtail operations. On the other hand, overdevelopment could needlessly commit productive resources and precious capital.

Achieving a balanced TSD strategy requires considerable planining and the analysis of widely varying alternatives, most likely under considerable scrutiny and severe schedule constraints.

The Solid Waste Projection Model (SWPM) system is an analysis tcol developed by Pacific Northwest Laboratory for Westinghouse Hanford Company specifically to address these issues. SWPM provides the ability to

- develop detailed projections of the volumes of wastes to be managed and the characteristics of these wastes

- characterize and evaluate the impact of alternative TSD technologies on facilities and operations

- assess the impact upon TSD cost and capacity requirements when waste volumes or waste characteristics vary

- track actual versus projected quantities of wastes, evaluating waste volume-reduction activities.

(a) The terms "treatment," "storage," and "disposal," as used here, do not necessarily correspond to the same terms used under the Resource Conservation and Recovery Act (RCRA). For example, storage, as defined by the model, could correspond to normal process holdup under RCRA. 


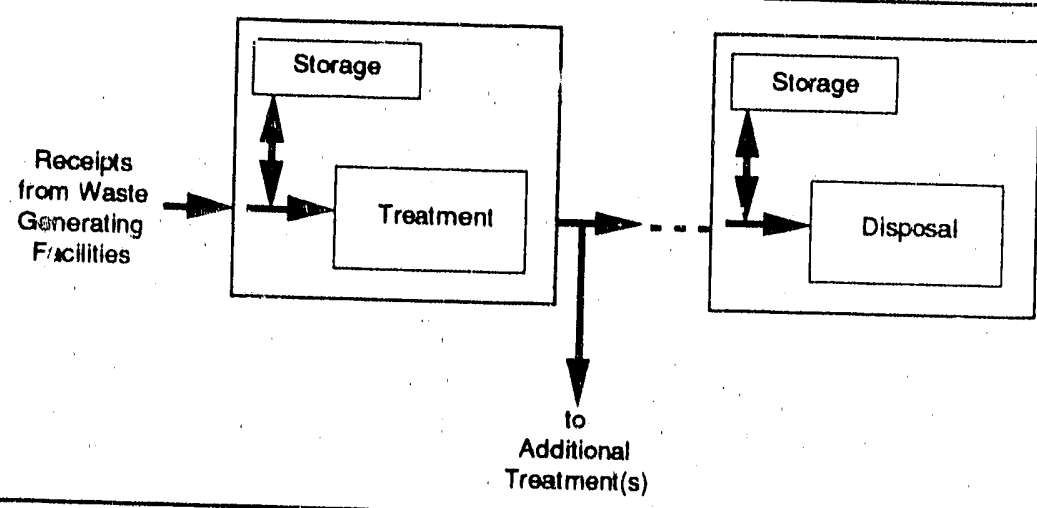

The Waste Management System Represented by the Model

\section{The System Being Modeled}

A generic representation of the solid waste management system modeled by SWPM is shown above. Solid wastes of a given type or class are received from a number of facilities and are distributed to various operations. Operations are defined as either treatments or disposals and are linked to other operations to represent a given waste management scheme. Waste volume may change as a result of some treatment operations. After treatment all wastes are redistributed to subsequent operations until a final destination is reached (disposal).

Each operation has an associated storage option to hold waste prior to treatment or disposal. All wastes eventually arrive at a disposal.

\section{The SWPM System}

The SWPM system uses a data-driven logistics model to provide detailed analyses of the generic system described above. The main module of SWPM is written in ADL, a proprietary modeling language developed by PNL. SWPM operates on an 80386 desktop computer in an $\mathrm{OS} / 2$ enviroriment and requires a minimum of 8 megabytes of RAM. 
As shown below, SWPM is supported by a database, which stores and maintains waste volume and characteristics projections, as well as the characterizations of the TSD operations and facilities. This information is passed from the database into two specially formatted electronic files, which match $\mathrm{ADL}$ syntax. These files, called the Reference Data Library, are loaded into the model's operating environment.

The key to operating SWPM is the graphical user interface, a tool which accesses the Reference D:ata Library and guides the user through the selection of data sets and analysis options, providing a complete and reproducible analysis case. Once this case has been specified, a run can be initiated from the user interface. The structured database and the discipline imposed by the user interface combine to provide consistency of approach and comparable results for all cases.

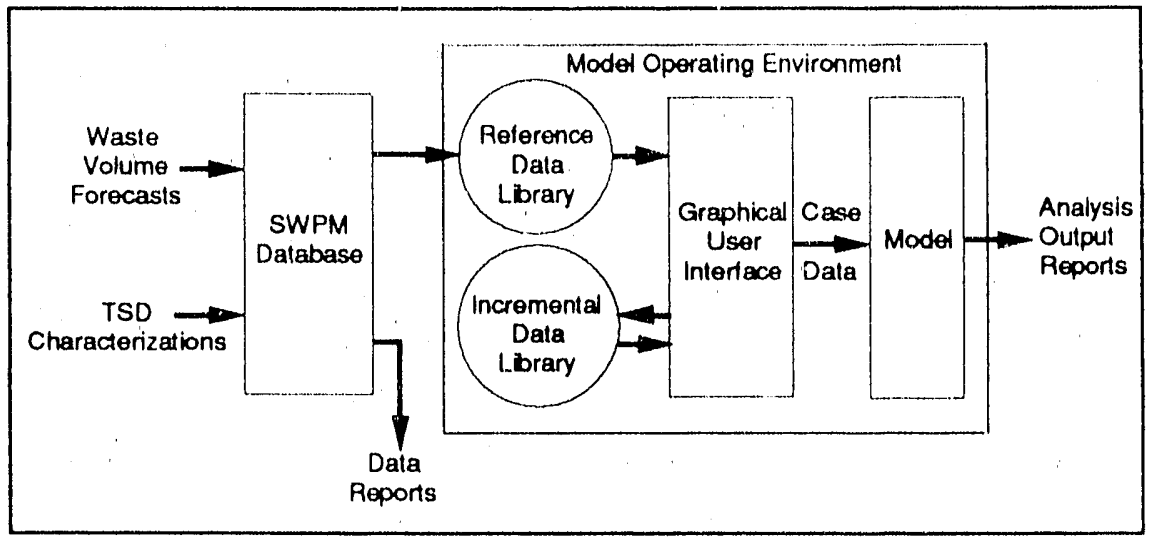

The SWPM System 


\section{The SWPM Database}

The SWPM database uses a relational structure developed specifically for long-term storage and maintenance of the data used in SWPM studies. The database contains the waste volume projections provided by the individual waste generators and the characterizations of the alternative TSD scenarios. The database also has custom data entry screens to facilitate the entry of large quantities of data.

As shown below, data describing solid waste volumes and characteristics must be entered, as well as the capacities of ail treatments and disposals, the unit costs for all operations, and the linkage instructions that describe the flow of waste through the system.

The database, with its structure and data entry screens, provides the ability to assemble and maintain many different waste volume projections and any number of TSD alternatives and to make large-scale changes to this important information quickly and easily. This flexibility allows systems analysts to characterize very different systems without making changes to the model or its accompanying software.

The primary information provided by the database is stored in the Reference Data Library. The library is then loaded into SWPM's operating environment.

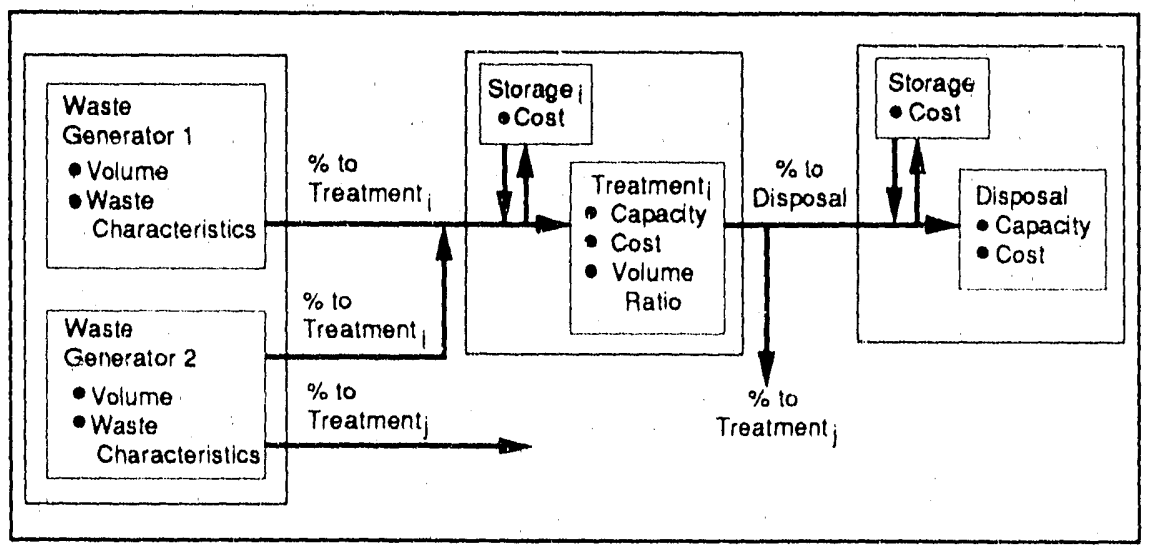

Data Used to Characterize a Waste Management System 
The data stored in the database are also useful in performing some. studies without the model. For instance, information such as the physical attributes of wastes or waste packaging can be reviewed using standard database reports. Also, database reports may be used to review, verify, and document the key information used in systems analyses.

\section{The SWPM Model User Interface}

The real key to the SWPM system is the model user interface, which allows the user to access and read the Reference Data Library and to construct a case for analysis. Using a system of simple "pull-down" menus, the user interface guides the analyst through the selection of a complete, logical, and syntactically correct data set for use in a model run. The user interface provides the capability to select only wastes or operations of interest for a case, yet ensures that sufficient data have been selected to initiate model runs.

Most important, the user interface provides system analysts with the opportunity to specify an alternative case without modifying the information stored in the database. Portions of the Reference Data Library can be modified, or components not included in the existing library can be created. Any modifications made to data using the user interface and/or newly added data are electronically stored in an incremental data library. Any such reference or incremental data library can be retrieved at any time through the user interface.

\section{The Model}

Once a data library has been prepared, a SWPM model run can be initiated through the user interface. The model executes without restricting access to the user interface and is essentially invisible to the user. The model is simple; yet because it is data-driven, it is capable of analyzing vastly different cases without software revisions. The model calculates and reports many factors critical to solid waste management, including the following:

- treatment, storage, and disposal costs

- disposal and treatment volumes by waste class or userdefined categories 
- treatment capacity requirements by waste class

- storage requirements by waste class or user-defined categories.

\section{Application of the SWPM System to Analyses}

Analysts can use the SWPM system and the reports generated from the database and model for numerous applications, such as the following:

- projecting waste storage capacity requirements and the cost of providing such storage space

- projecting waste treatment or waste disposal volumes and the resulting capacity requirements, utilization, and costs

- developing budgetary planning information

- assessing system cost and capacity impacts of now technologies or alternative operating strategies

- assessing system cost and capacity impacts of varying waste receipts

- developing and assessing stored waste retrieval schedules

- assessing the credibility of waste volume forecasts.

\section{Important Factors in Developing Solid Waste Projections}

Although conceptually straightforward, development of reliable projections will be neither simple nor inexpensive. Substantial quantities of data are required to provide a reasonable basis for the projections, and the required analyses must be carefully defined and carried out to ensure applicability to the specific needs. Gathering, checking, and integrating the necessary data are time-consuming and challenging activities. 
Careful planning before actual model runs will optimize data development efforts and will maximize the effectiveness of SWPM application. The following are several factors important in the timely, efficient and cost-effective development of reliable, useful projections:

- The representation of the waste management aystem, which provides the basis used for projections and analyses, must be realistic. In many cases, external (e.g., regulatory) or internal (e.g., policy or planning) requirements must be reflected in the system. The requirements for handling and managing different waste types must be clearly understood.

- The data used to characterize the system must be carefully developed, reviewed, documented, and controlled throughout the life of the modeling system. Whimsical data will result in misleading conclusions about the need for specific waste management capabilities.

- Projections should be scoped at the appropriate level of detail; insufficient detail will provide information which does not clearly answer the questions involved. Too much detail will obscure the important information and make analyses unwieldy.

- Because of the uncertainty and variability involved with much of the data, results must be carefully interpreted. Some "what-if" scenarios should be analyzed to identify the specific input parameters to which the results are most sensitive. 


\section{Conclusion}

Achieving a balanced solid waste management strategy requires considerable planning and the analysis of widely varying alternatives. Because solid waste management is becoming a critical, highly visible activity, the evaluation of these alternatives will most likely occur under considerable scrutiny and severe schedule constraints.

The SWPM system, built around a data-driven logistics model, provides a means of characterizing many alternative systems and of quickly assessing the relative performance of each. Using the SWPM system, reliable projections of solid waste treatment, storage, and disposal operations and facilities can be made. These projections can then be used to make decisions regarding the waste management system configuration, operation capacities, facility capabilities, and operating strategies. 


\section{Further Documentation}

Five additional documents provide detailed instructions in the use, maintenance, and application of each of the components of the SWPM system.

Title

Database User's

Guide

Model User's

Guide

Database

Technical

Reference Manual

Model

Technical

Reference Manual

System

Administration

Manual
Description

User's instructions for data entry, maintenance, and reporting

User's instructions for model operation and execution

Deyelopment and maintenance reference manual for database software and utilities

Development and maintenance reference manual for model software and applications

Instruction fur lorig-term system administra ion, mantenance, and configur ation management
Primary Audience

System

Administrator

Analyst

Database

Specialist

Software

Engineer

System

Administrator

\section{Contacts}

For further information about the application or operation of SWPM, contact:

Dennis Stiles, (509) 376-4154

PNL project manager

SWPM application and analytical support

Greg Holter, (509) 376-7696

PNL senior analyst

analytical support

Steve Shaver, (509) 376-4258

PNL task leader

model operation and model user support 

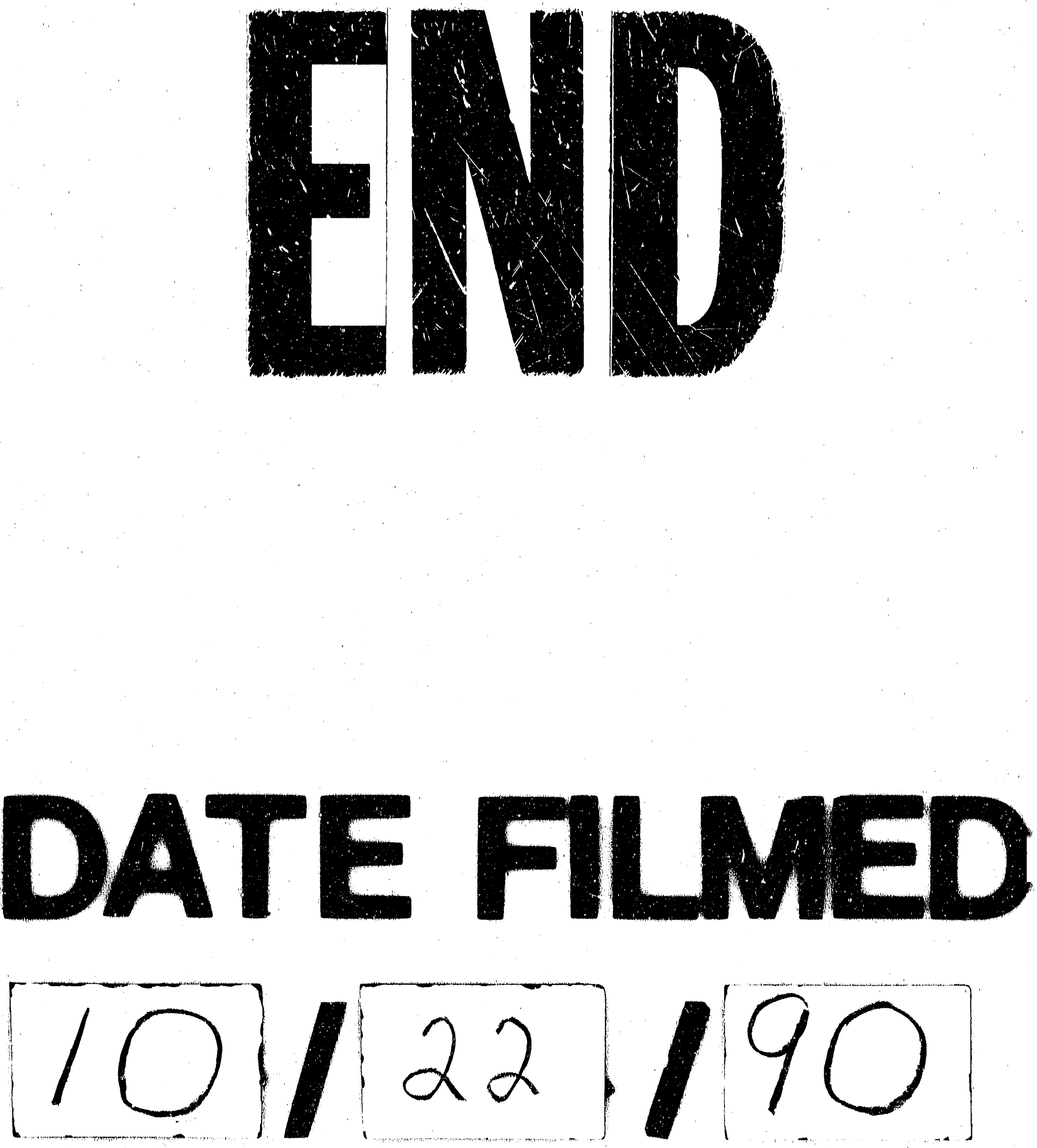